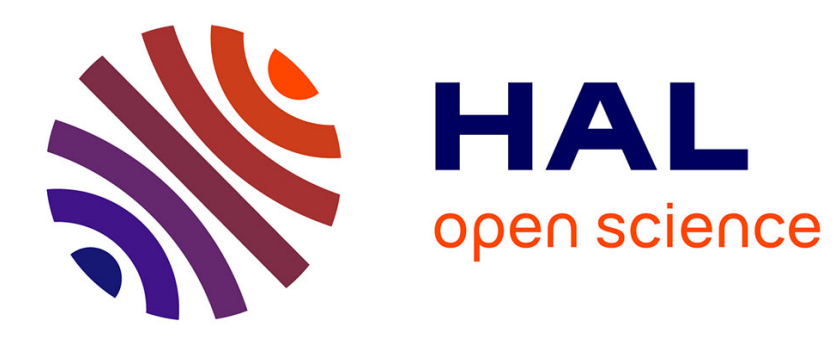

\title{
Propositions sur la théorie de la police
}

Fabien Jobard

\section{To cite this version:}

Fabien Jobard. Propositions sur la théorie de la police. Champ Pénal, 2012, IX, http://champpenal.revues.org/8298. hal-00744202

\section{HAL Id: hal-00744202 \\ https://hal.science/hal-00744202}

Submitted on 22 Oct 2012

HAL is a multi-disciplinary open access archive for the deposit and dissemination of scientific research documents, whether they are published or not. The documents may come from teaching and research institutions in France or abroad, or from public or private research centers.
L'archive ouverte pluridisciplinaire HAL, est destinée au dépôt et à la diffusion de documents scientifiques de niveau recherche, publiés ou non, émanant des établissements d'enseignement et de recherche français ou étrangers, des laboratoires publics ou privés. 


\title{
Champ pénal/Penal field
}

Nouvelle revue internationale de criminologie

\author{
Vol. IX | 2012 : \\ Varia
}

Actes de colloque/Conference Proceedings - L'œuvre singulière d'un penseur cosmopolite : Hommages à Jean-Paul Brodeur

\section{Propositions sur la théorie de la police}

\author{
FABIEN JOBARD
}

\section{Résumés}

Cet article revient sur les débats que Jean-Paul Brodeur avait développés durant au moins deux décennies autour de la place de la force dans la définition et la théorie de la police. L'auteur expose d'abord le projet théorique de The Policing Web, son dernier ouvrage, et la place qu'occupe dans celui-ci « l'illégalisme policier », qui constitue la pierre fondatrice de la théorie de la police avancée par Jean-Paul Brodeur. Il esquisse ensuite une approche sociologique de cet illégalisme policier, en montrant pour quelles raisons la méthode retenue par Jean-Paul Brodeur ne pouvait être qualifiée de sociologique, avant de formuler une " théorie sociologique de la police », fondée sur la notion de souveraineté. L'emploi de cette notion le conduit à préciser son acception, avant de formuler une proposition de « théorie politique de la police».

This article revisits several arguments that Jean-Paul Brodeur developed for at least two decades on the role of the use of force in the definition and a general theory of policing. The author examines the theoretical project that characterizes The Policing Web, his last book, and assesses where "legal lawlessness", the touchstone of the theory of policing put forward in the book, fits. He then outlines a sociological approach of legal lawlessness, considering that Jean-Paul Brodeur's chosen method could not be considered a sociological one. At that point, a "sociological theory of policing" will emerge, based on the concept of sovereignty. After having clearly defined this concept's accepted meaning, a "political theory of policing" is formulated.

\section{Entrées d'index}

Index de mots-clés : Jean-Paul Brodeur, force, illégalisme policier, souveraineté, théorie de la police, violence policière

Index by keyword : Jean-Paul Brodeur, force, police legal lawlessness, police violence, sovereignty, theory of policing

\section{Texte intégral}




\section{Introduction}

Mon intervention est l'occasion, forcément attristée, de revenir sur les débats que Jean-Paul Brodeur avait développés durant au moins deux décennies autour de la place de la force dans la définition et la théorie de la police. Ces débats nous ont réunis périodiquement, comme en témoignent diverses publications ${ }^{1}$, et c'est tout naturellement que mon hommage empoignera, à nouveau, la question de la violence policière - dans une actualité française marquée comme elle l'est parfois par des affaires de nature diverse touchant l'usage de la force par la police ${ }^{2}$.

2 Jean-Paul Brodeur est revenu très abondamment dans son ouvrage posthume The Policing Web sur la place de la force physique dans le métier policier, notamment dans le chapitre 4 consacré à la théorie de la police. Ce chapitre est tout entier consacré à la réfutation puis l'amendement du PUFP (Police Use of Force Paradigm) proposé par Egon Bittner. Pourtant, dans l'ouvrage d'hommage à Dominique Monjardet, le sociologue français de la police décédé en 2006, Brodeur estimait qu'on avait « fait trop grand cas » de cette question de la force physique dans la définition de la police (Brodeur, 2008a). Je prends donc ici la précaution exprimée par Jean-Paul comme une prétérition, et pour ce qu'elle est en réalité : une invitation.

Pour honorer cette invitation, j'exposerai tout d'abord le projet théorique du Policing Web et la place, dans ce projet, du legal lawlessness, "l'illégalisme policier $»^{3}$, qui constitue la pierre fondatrice de la théorie de la police avancée dans le Policing Web. J'esquisserai ensuite une approche sociologique de cet illégalisme policier, étant entendu que, comme j'essaierai de le montrer, la méthode retenue par Jean-Paul Brodeur ne pouvait être qualifiée de sociologique. Nous serons ensuite en mesure de formuler une " théorie sociologique de la police ", fondée sur la notion de souveraineté. L'emploi de cette notion nous obligera à préciser son acception, avant de formuler une proposition de «théorie politique de la police » et de conclure.

\section{I - Jean-Paul Brodeur : une nouvelle approche théorique de la police}

Dans son traité sur la police, The Policing Web, Jean-Paul fait part de sa ferme résolution à to provide a new definition of policing and (to) introduce some of the elements of a theory of policing (6). Sa definition builds on the notion of legally using means prohibited to others (6). Elle est délivrée dans les dernières pages de son chapitre consacré à la théorie de la police (chapitre 4, 130) :

\footnotetext{
Policing agents are part of several connected organizations authorized to use in more or less controlled ways diverse means, generally prohibited by statute or regulation to the rest of the population, in order to enforce various types of rules and customs that promote a defined order in society, considered in its whole or in some of its parts.
}

D'emblée, on relève que si l'une des ambitions déclarées de Jean-Paul Brodeur est d'interroger the nature of theory (Policing Web, 7), le produit de son investigation est une définition, mais pas une théorie ; comme si la difficulté première était non pas de délivrer un ensemble de propositions déduites d'observations établies, mais d'embrasser en une seule formulation à la fois la spécificité et l'étendue de l'institution policière. Car le vrai problème auquel Policing Web est consacré n'est pas, en réalité, la police comme objet théorique propre à un ensemble de savoirs (les sciences sociales, la philosophie ou autres, qui feraient de sa théorie une théorie sociologique, une théorie politique ou autre). Le problème de Brodeur, qu'il présente comme le challenge de Policing Web (8) est l'unité de la police, unité qui se trouve contredite par l'éclatement et la diversité que l'institution et ses agents opposent 
immédiatement à l'observateur. Sa définition, qui multiplie le recours aux pluriels : policing agents ${ }^{4}$, several connected organizations, a pour fonction de saisir l'ensemble des activités et des statuts policiers, de l'inspecteur en charge d'affaires financières ou de contrôle de l'hygiène au policier du coin de la rue, en passant par l'agent affecté au maintien de l'ordre et celui employé à des missions de renseignement.

Le lecteur aura cependant la surprise de constater que Jean-Paul Brodeur se dit insatisfait de sa théorie de la police, à laquelle il trouve des antinomies or discordances (...) that cannot be entirely resolved (Policing Web, 6).

7 La première antinomie réside dans la discordance entre " visibilité » (openness) et « secret ». En effet, si Brodeur poursuit une théorie moniste de la police ou, comme il l'affirme dès les premières pages de Policing Web, une théorie de la police completeness (Policing Web, 9-10), il déplore de buter sur une réalité duale de la police. De police, dit-il, il en est en réalité deux : une visible et une secrète, et ces deux polices ne se recouvrent jamais l'une l'autre ${ }^{5}$.

La deuxième antinomie (which is specific to the theory of policing) repose sur le fait que la police est une tainted occupation (Bittner 2001/1974), dans la mesure où le travail de police revêt certaines des caractéristiques de ses cibles et s'entache de suspicion $(2001,302)$; et cette face obscure de la police contraste fortement avec la police qui, dans son essence, est une profession qui opère dans un régime de légitimité (Reiner, 1992), de consentement (Skogan, Frydl 2004), ou de confiance (Manning 2003).

Je défendrai ici l'idée que les discordances invoquées par Jean-Paul n'en sont pas.

Les phénomènes qui l'amènent à poser ces discordances (le couple visibilité/invisibilité d'une part, le couple confiance/hostilité d'autre part) appellent un réexamen sociologique, qui permettra une reformulation de la position de Jean-Paul Brodeur. Cette reformulation consiste en une tentative de théorie sociologique de l'illégalisme policier. Une certaine ambiguité, nous l'avons dit, plane sur la démarche de Brodeur, qui souhaite produire une «théorie » de la police, et même une théorie «moniste » de la police, mais qui n'avance qu'une définition de la police. Dans Social Theory and Social Structure, Merton estimait qu'en sciences sociales une théorie était une proposition ou un ensemble de propositions liées entre elles par une chaine de déductions ; par exemple la production d'idées révolutionnaires engendrée par la croissance de l'écart entre le salaire obtenu et le salaire espéré. Rien de tel chez Brodeur, qui s'en tient à une définition de son objet et non à une théorie de cet objet. Or, nous défendons ici que c'est bien l'absence d'une théorie qui l'amène à voir ces discordances comme invalidations partielles de sa définition.

Notre construction théorique partira du point d'arrivée de Jean-Paul : ce qui définit ou en tout cas caractérise la police est son « illégalisme légal », c'est-à-dire sa faculté de se voir autorisée par la loi à commettre des actes en infraction à la loi commune. À partir de ce point d'accord, tout nous sépare.

\section{II - Dualité des espaces sociaux et des circonstances}

L'oxymore legal lawlessness désigne l'ensemble des actes commis en infraction à la loi commune en vue du respect plus général de celle-ci ; par exemple le fait de s'engager dans une voie en sens interdit pour mieux appréhender un suspect en fuite, ou d'écouter des conversations privées au moyen de dispositifs plus ou moins 
sophistiqués d'interception, de captation, de sonorisation, etc. ${ }^{6}$. Brodeur considère l'illégalisme policier comme donné par la loi, comme immanent à l'activité policière. Le Code de procédure pénale canadien, relève-t-il, consiste en un grand catalogue des infractions que les policiers peuvent commettre sans crainte (Policing Web, 130-131).

En réalité, l'examen empirique de cet illégalisme légal montre que, bien qu'établi par la lettre du Code, il se définit par le fait qu'il n'est jamais donné une fois pour toutes : il est mouvant, instable, fragile, soumis à l'assaut réitéré de la société et des juges, ces derniers particulièrement inquiets de voir prospérer en marge de l'ordre normatif ordinaire un espace soustrait à leur emprise (que Jean-Paul appelle « extra-légal » dans le Policing Web). C'est ce que la doctrine juridique désigne, entre autres exemples ${ }^{7}$, par la notion d'excuse absolutoire, toujours soumise à l'appréciation in concreto des juges. In concreto : non pas dans l'exégèse du Code, mais dans l'examen scrupuleux des circonstances concrètes de l'intervention policière. Ce sont donc d'abord les juges qui qualifient la nature légale ou illégale de l'illégalisme policier, qui font basculer le geste policier dans le régime commun ou dans la zone grise de l'illégalisme légal.

Comprendre l'illégalisme légal dont jouiraient les policiers exige donc de faire l'examen du sort que les juges réservent aux interventions policières sur lesquelles ils sont appelés à se prononcer. Ce que cet examen révèle, c'est que l'illégalisme n'est légal que sous certaines conditions. Il y a en effet un espace social de l'illégalisme policier, que je propose d'examiner en quelques mots.

Prenons une intervention policière, qui en 1991 mit des policiers d'un service judiciaire de la banlieue parisienne aux prises avec un trafiquant d'héroïne présumé, M. Selmouni. Cette interaction a amené le gouvernement français à se voir condamné en 1999 pour " torture » par la Cour européenne des droits de l'homme, condamnation qui n'avait été jusque lors que prononcée à l'encontre du gouvernement turc (Uildriks, 1999 ; Rodey, 2005, 406). Même une fois cette condamnation prononcée par les instances européennes, les policiers ne se virent condamnés par la justice française qu'à des peines d'emprisonnement avec sursis (sur cet écart significatif, voir Cahn, 2007). Les juges européens ont, eux, estimé que M. Selmouni avait été battu durant les quatre jours de sa garde à vue, en plus d'être exposé à des traitements humiliants et dégradants ; épisode qui lui valut, toujours selon les juges de la Cour européenne, la perte de son œil gauche.

16 À l'examen de cette affaire, les juges français exposèrent une position tout à fait intéressante.

Considérant que dans l'absolu la parole d'un policier, a fortiori celle d'un officier de police judiciaire, est plus crédible que celle d'un trafiquant de drogue ; que toutefois ce postulat est fragilisé, voire ébranlé lorsque les déclarations des délinquants sont confortées par des éléments extérieurs tels que des constatations médicales ;

Considérant qu'il n'est pas sans intérêt de noter que Selmouni n'ayant jamais été placé en garde à vue ne pouvait utiliser son expérience en la matière pour échafauder un montage mensonger ; que Selmouni n'a pratiquement jamais été assisté d'un conseil dans la procédure relative au trafic de stupéfiants ; que l'ensemble de ces éléments conduit la cour à se convaincre de ce que la rébellion alléguée a été imaginée par les mis en cause pour justifier l’importance et la localisation des hématomes et lésions présentés par le gardé à vue.

Qu'est-ce que l'illégalisme policier ? Cette décision judiciaire nous aide à le comprendre. Des éléments d'attestation de l'atteinte sont requis par les juges pour convertir avec succès une allégation en fait constaté (sur cette conversion, voir Jobard, 2003). Des éléments matériels comme un certificat médical ou des 
témoignages par des tiers sont des pièces tout à fait importantes dans ce processus de certification, mais elles ne sont en aucun cas suffisantes ${ }^{8}$. Car si l'on s'en tient à l'argumentaire déployé par les juges de la cour d'appel de Paris en 1999, s'ajoute à ces conditions extérieures à la victime putative une condition qui tient à sa constitution même : celle-ci ne peut être crédible que si elle ne relève pas de la clientèle policière, de la police property évoquée par Lee (1981) dans son article séminal. On peut même dire que les conditions du succès de la conversion de l'allégation en fait avéré reposent sur sa virginité pénale : les juges distinguent le monde de ceux qui savent (mentir) et le monde de ceux qui ne savent pas. Dans un cynisme sans doute involontaire, les juges notent que le plaignant n'avait pu voir d'avocat ${ }^{9}$, ce qui à leurs yeux confirmait sa sincérité. Eût-il échangé avec un avocat, son témoignage en eût été vicié. Le juge trace une ligne de démarcation très nette entre les êtres : les êtres sains (et crédibles), les êtres souillés (et inaudibles). Si l'on étend au judiciaire les termes de la sémiotique des sciences et des techniques, on dirait que le plaignant, pour être entendu, pour être crédible, doit être conforme à un " script » d'airain (Akrich, 1992), établi par les juges et aussi intransigeant que le script d'un détecteur de pièces métalliques qui bipe ou ne bipe pas lorsque passe un voyageur entre ses parois (Jobard, Linhardt, 2008). Selmouni ne gagne donc pas la crédibilité de son témoignage par la maladresse des policiers et le caractère exorbitant des violences exercées contre lui. Ces deux conditions, nécessaires, ne sont pas suffisantes. Sa force probante, il l'acquiert aussi par sa qualité d'homme commun. Par sa qualité d'homme vierge de toute corruption par un contact antérieur avec la machine judiciaire et les ruses qu'elle enseigne. Pas sa qualité d'homme sans qualités pénales.

Que l'on décrive les chances de succès du récit de la violence alléguée par les notions de script de la sociologie des sciences et des techniques ou par les notions anthropologiques du pur et de l'impur (Douglas 1966), on voit se dessiner l'espace particulièrement exigu dans lequel une violence policière illégitime est attestée comme telle par la société ${ }^{10}$. Faute de pouvoir entrer dans les détails de sa constitution, on peut se contenter de dessiner cet espace de recevabilité de la violence policière comme une sorte de quadrilatère dont les sommets sont liés les uns aux autres de manière interdépendante. Le premier sommet est celui, on vient de le voir, de la pureté pénale. Le deuxième est celui de la taille de l'atteinte : l'atteinte portée doit être de taille notable, remarquable. Le troisième est celui de l'attestation. Matérielle ou testimoniale, elle est soumise à la même exigence de pureté que celle qui est attendue de la victime putative. Si le témoin est lié le moins du monde au plaignant, ou s'il ne présente pas la fiabilité ou la pureté exigée par le script judiciaire, sa parole est disqualifiée, elle ne peut plus faire convoquer à son avantage d'opérateurs de factualité (Dulong, 1997, 1998). Le quatrième est celui des circonstances de l'atteinte. Nécessaire à la conversion du fait allégué en fait établi est la conviction que les policiers ont agi hors tout danger ou menace pesant sur eux. Notons ici que, plus que pour les autres, cette propriété du quadrilatère n'est pas exempte d'intervention policière. Dans un rapport sur les fautes disciplinaires au sein de la Police nationale, le directeur de l'Inspection générale de la police nationale ${ }^{11}$ relevait que la proportion des faits établis oscillait selon les années entre $7 \%$ et $20 \%$ des cas allégués dans les cas où le requérant n'était pas lui-même visé par une plainte déposée par les policiers, et entre $0,6 \%$ et $7 \%$ des allégations lorsque le requérant était visé par une plainte déposée par les policiers. Le directeur ajoutait alors :

Lorsque la force n'est pas nécessaire, ou qu'elle est dépassée, la violence devient

illégitime. Elle peut alors s'abriter derrière un rapport d'outrage, de rébellion ou de violence à agent. C'est à ce stade que se situe la difficulté d'établir la vérité. (Le Doussal, 1988, 41) 
Sans aller plus loin dans le détail explicitant les contraintes pesant sur la conversion de l'allégation en fait établi, nous constatons que c'est dans les espaces sociaux où la police fait le plus usage de la force que cet usage est le moins susceptible d'être porté devant les tribunaux. Dans les zones urbaines reléguées, la concentration est telle [des jeunes hommes déjà condamnés ou, plus encore, des jeunes hommes « connus des services de police » et enregistrés dans les fichiers de police comme ayant été au moins une fois interpellés], qu'il est bien difficile de trouver des témoins sans qualités pénales. $\mathrm{Ni}$, compte tenu du degré de ségrégation sociale dans ces zones (Lagrange, 2009), de tiers non impurs. Les zones de forte tension, les zones dans lesquelles la police est le plus susceptible d'employer la force sont celles où la légitimité de l'emploi de la force policière a le moins de chances d'être portée sur la place publique. Silence et violence forment dans ces espaces sociaux comme les deux faces d'un même ruban de Möbius.

On le voit donc : la dualité entre visibilité et secret, sur laquelle Brodeur estime que bute sa " théorie » de la police, est en réalité, dans la réalité empirique du devenir des événements de violence, constitutive de la police. La police n'est pas un organisme qui se scinde en deux organismes étanches l'un à l'autre, la haute et la basse police (Brodeur 1983) ; elle est constitutivement duale : visible ou invisible selon les circonstances, selon les lieux, selon les protagonistes. La dimension sale, suspecte, tainted, de la police, dont Brodeur dit qu'elle gêne sa théorie de la police, affecte à la fois la police et la société dans laquelle la police intervient. La police garde une capacité d'entacher la réalité et de salir ses adversaires par le récit qu'elle fait de son intervention. Et toutes les parties de la société ne baignent pas dans une égale lumière, si bien que les actes que la police commet sont à leur tour inégalement susceptibles de relever du regard public.

On le voit, la notion de « visibilité » doit être rapportée au régime juridique de la publicité, par exemple de la publicité des audiences, qui entraîne l'égalité des armes, etc. Justice est rendue lorsque les parties ont un accès équitable à elle, lorsque les charges, les preuves, les motivations sont produites devant le public, lorsque tout simplement l'intervention policière fait l'objet d'une dramatisation (Manning, 2010, x) qui amène le public à être juge ou, ce qui revient au même, les juges à rendre compte devant le public de la manière dont ils jugent ${ }^{12}$. Or, la sociologie de l'usage de la force par la police montre un espace incroyablement exigu de réception des allégations de violence ; un espace inéquitable, qui astreint la plus grande partie des allégations à ne jamais passer le seuil de la publicité, faute d'être entendues, faute d'être audibles.

La question de la publicité de la police, où se mêlent l'exigence de visibilité et le constat attristé de l'aspect tainted du métier policier, est cruciale pour une théorie de la police. L'obscurité ou l'ombre ne sont pas des obstacles au déploiement d'une théorie de la police, comme semble le regretter Jean-Paul Brodeur, mais constituent bien la pierre de touche d'une théorie politique de la police, comme nous allons le montrer maintenant.

\section{III - La place de la souveraineté dans la théorie de la police}

Dans les espaces sociaux de relégation, là où l'intervention policière est la plus impérieuse, on observe que tant que les policiers ne commettent pas d'excès dans leur déviance (caviarder de manière trop rudimentaire des procès-verbaux, se tromper de manière flagrante sur l'identité sociale de l'adversaire, tirer sur un 
individu déjà menotté, etc.), il n’y aura pas de sanction de la déviance policière (sans même se prononcer sur l'échelle des sanctions prononcées). La définition de Jean-Paul Brodeur est fondée sur un implicite continuum des moyens policiers : authorized to use in more or less controlled ways diverse means.

Cette observation ne correspond pas à la réalité empirique du rapport des policiers à la loi. Il y a un rapport déviant à la loi, qui peut être sanctionné par le juge, et il y a un illégalisme qui ne sera pas sanctionné, car tenu hors du regard du juge. Il y a donc comme deux polices. Non pas deux institutions policières comme la haute police et la basse police, non pas deux pratiques policières antagoniques comme l'îlotier et l'agent sous couverture, mais deux modalités du policing selon les circonstances et les espaces sociaux de l'intervention policière. Il est des espaces sociaux dans lesquels l'intervention policière ne fera tout simplement l'objet d'aucun recours, dans lesquels la police, donc, est souveraine. Dans ces espaces ou ces circonstances, la police se substitue au droit : elle fait la norme, étant entendu qu'il n'est pas de droit sans sanction. La police relève dans ces espaces ou dans ces circonstances d'une théorie décisionniste du droit, dans laquelle (à la différence du normativisme) ce n'est pas une norme suprême (une constitution, un Bill of Rights, un Code...) qui fait le droit, mais le geste individuel de celui qui tranche sans risque d'être contredit. Cette théorie décisionniste du droit est énoncée ainsi par le juriste allemand Carl Schmitt (1934, 20) :

Souverain est celui qui décide en et de la situation exceptionnelle.

Rappelons ici la double portée de cette définition de la souveraineté. Souverain est celui dont la décision n'est susceptible d'aucun appel dès lors qu'elle est prise en situation exceptionnelle. Souverain est, aussi, celui qui a la faculté de décider que l'on bascule en situation exceptionnelle et que le droit ordinaire est alors suspendu, sans effet. C'est dans cette deuxième acception que l'on entendra la notion de police discretion (l'un des plus anciens concepts de la sociologie de la police - cf. Klockars, 1985), que nous avons illustrée par l'amer constat formulé il y a 25 ans par le directeur de l'IGPN d'alors, lorsqu'il constatait la capacité de réécrire la situation post hoc. Les policiers jouissent, dans certains espaces sociaux, de cette faculté de priver leur vis-à-vis de la possibilité d'accéder à la justice, et ainsi de faire basculer la situation dans le régime de l'exception. Il existe des arènes particulières, dans lequel le droit est autre.

Cette disposition si particulière du rapport de la police à la souveraineté a plusieurs conséquences. La plus évidente concerne l'État, la puissance publique : dans certains espaces sociaux, ou dans certaines circonstances, la situation d'exception manifeste dans tout son éclat l'essence de l'autorité de l'État (Schmitt, 1934, 19). Dans ces espaces ou ces circonstances, la norme juridique ne se suffit plus à elle-même, et la puissance publique est appelée à se manifester dans l'éclat tranchant de la décision sans appel.

Mais la conséquence théorique la plus saillante du décisionnisme révélé par l'abstention de la justice pénale face à l'illégalisme policier concerne non pas la puissance de l'État mais, si l'on entend la notion de souveraineté dans le sens que lui confère Schmitt, dans le rapport de la police au politique. Avant d'évoquer cette dimension, il faut signaler, préventivement, les dangers auxquels expose la notion de souveraineté appliquée à la police, tant le décisionnisme schmittien a généré ces derniers temps un certain nombre de conceptions absolutistes sans aucun rapport avec la réalité des sociétés dans lesquelles nous vivons.

\section{IV - Une théorie sociologique de la police (ou : ce qu'une théorie}




\section{décisionniste de la police n'est pas)}

Toute notre démonstration se fonde sur l'idée selon laquelle la police n'est pas la même dans les différents espaces qui forment une société. L'idée est ancienne et banale (Reiss, Bordua, 1967). Ce qui surprend, c'est que ses conséquences théoriques ont été comme inaperçues, et d'abord ses conséquences sur la place du droit dans la police. Comme le souligne Pierre Favre $(2010,1237)$ :

Les sciences sociales ont souvent de la réticence à admettre le caractère déterminant de l'existence de forces policières - au sens large - dans la pérennisation de l'ordre social et plus encore dans sa production. Les sociologues font ainsi valoir que l'intériorisation de l'ordre est maintenant acquise dans les sociétés avancées et que le recours à la force n'est plus nécessaire. Ils font remarquer à juste titre que l'intervention policière violente est l'exception, mais cela les amène à effacer de leurs analyses toute trace de l'usage policier de la force.

Lorsque Brodeur avance une définition de la police centrée sur l'illégalisme policier, il exclut du champ de cette définition le fait que le droit, loin d'être un bien universel, reste un bien socialement exclusif - comme si ce qui relevait d'une sociologie de la mise en œuvre différentielle du droit par la police (la sociologie du law enforcement au sens strict, telle qu'engagée par Black, 1973) relevait d'un domaine particulier de l'investigation sociologique, mais n'avait plus sa place dans une théorie de la police. Brodeur relève que la police est essentiellement un selective policing (Policing Web, 119), mais cette considération laisse inaltérée sa définition de la police, dont il veut protéger la completeness, tout en la sachant menacée par la dualité... L'approche par le décisionnisme schmittien est non seulement la conséquence théorique d'une sociologie de la police qui prend le droit pour point d'appui, le droit dans son existence concrète, mais elle permet de surcroît de rendre justice à la dualité et au caractère tainted de la police.

Cette théorie décisionniste procède d'une géographie sociale sous-jacente. Dans la plus grande partie de la société, la police est soumise au droit ordinaire ; dans des fractions plus particulières, elle s'en émancipe. Il ne s'agit donc pas, sous prétexte de recourir à Schmitt, un des juristes au centre d'un scandale permanent dans l'histoire intellectuelle (Müller, 2003), de faire de l'institution policière le vecteur d'un nouvel ordre mondial marqué par « l'exception permanente » ou le basculement de nos sociétés dans un nouvel âge bio-politique marqué par le triomphe du pouvoir souverain sur la vie nue, s'il nous est permis de résumer ainsi à grands traits l'éreintante glose engendrée ces deux dernières décennies par certains lecteurs de Schmitt. Du monde, il n'est pas question ici, mais d'une société très concrète et de ses fractures très concrètes ; l'enjeu est bien de produire une théorie de la police, ancrée dans la sociologie de ses pratiques (voir pour une démarche comparable Fischer, Spire, 2009, 13-14; par ailleurs mes critiques à l'égard de « la glose » rejoignent très largement celles de Fischer, 2011).

Par ailleurs, il n'est pas non plus question de pouvoir absolu, sous prétexte de souveraineté. Les pouvoirs concrets de la police dans les zones de souveraineté se trouvent pris dans le quadrilatère que nous avons dessiné. Les policiers doivent toujours jauger de la nature de celui qui leur fait face, de l'éventualité de témoins crédibles et de la taille de l'atteinte qu'ils sont susceptibles d'infliger. Leur souveraineté est limitée par l'anticipation de l'accueil que leurs collègues, puis les juges, réserveront au récit qu'ils seront capables de produire après l'intervention. Cette contrainte limite fortement l'ampleur de ce qu'ils peuvent faire (Manning, 1978 ; Jobard, 2005), et indexe toujours l'action policière à la nature du milieu dans lequel les policiers interviennent. Le policier, le streetcorner politician de William Ker Muir (1977), est un acteur politique en ce qu'il jauge, devant l'interaction à venir, ce qui lui est politiquement possible de faire, et ce qui ne lui est pas, politiquement, 
possible de faire (voir dans un sens comparable les travaux de Josiah McHeyman sur les agents de la frontière mexicano-américaine, p. ex. McHeyman, 2000). Le policier mesure le mandat politique que lui confie la société au moment même où il s'engage dans l'interaction. Plus exactement : il estime si les conditions politiques sont réunies qui permettraient, s'il le souhaite, d'engager un acte de souveraineté, ou si elles ne le sont pas.

C'est en ce sens qu'il faut comprendre la police discretion mise en avant par les sociologues nord-américains depuis des décennies. Parce qu'il est un pouvoir qui inclut celui d'user de la force, jusqu'à donner la mort, et parce qu'il est un pouvoir devant lequel, dans certaines circonstances, tous les autres pouvoirs s'inclinent, ce pouvoir discrétionnaire touche à la souveraineté : c'est un pouvoir politique. Car, comme nous l'avons suggéré supra, ce n'est pas seulement le remplacement du droit par la puissance pure de l'État qui est en jeu, mais la notion de politique.

\section{V - Une théorie politique de la police}

La compréhension empirique du destin des actes d'illégalisme commis par les policiers nous permet de dégager un espace de souveraineté, dans lequel les policiers peuvent se mouvoir. Quelles implications ce constat entraîne-t-il ? Quelle est la valeur ajoutée de nos observations ? S'agit-il seulement de remplacer une notion (la police discretion ou la legal lawlessness) par une autre (la souveraineté) ? Le détour par la théorie décisionniste de Schmitt est décisif car il ouvre des perspectives qui permettent d'insérer la police dans l'édifice plus large de la " théorie politique », domaine qui embrasse à la fois la théorie de la justice de Rawls ou Hart (voir à ce sujet Manning, 2010) mais aussi la théorie de l'État ou plus largement de la communauté politique, la polity.

Relevons à ce sujet, s'il fallait convaincre de la nécessité de penser la police comme élément de la théorie de l'État, que les cours de Pierre Bourdieu sur l'État, qui viennent tout juste d'être édités (Bourdieu 2012), font d'un habile tour de passe-passe s'évaporer la police et, plus largement, la coercition, de la définition de l'État. Dans un tel contexte, le terme " police » n'apparait pas au sommaire des 660 pages du cours et, dans ses 9 occurrences tout au long de l'ouvrage, le terme est le plus souvent cité comme métonymie des appareils répressifs d'État puisqu'il est cité indifféremment aux côtés du terme " armée » voire de l'ensemble des institutions mandatées pour garantir l'ordre $(22,215,239,315,327)$. Voici comment s'opère l'opération de dissimulation de la force physique et de la police dans la polity contemporaine :

J'ai fait, il y a déjà plusieurs années, une addition à la célèbre définition de Max Weber qui définit l'État comme le 'monopole de la violence légitime', que je corrige en ajoutant : 'monopole de la violence physique et symbolique'; on pourrait même dire : 'monopole de la violence symbolique légitime', dans la mesure où le monopole de la violence symbolique est la condition de la possession de l'exercice du monopole de la violence physique elle-même. (Bourdieu 2012, 14)

Or, si l'on déroule, comme je prétends le faire ici, le fil de l'illégalisme policier jusqu'au bout, pour découvrir la notion de souveraineté, on voit bien que se joue autre chose que la violence physique comme appendice de la « violence symbolique ». Car, ce à quoi conduit la notion de souveraineté telle que portée par l'action quotidienne de la police, c'est à une compréhension plus approfondie de la dimension politique de la police. La plupart des auteurs ont, comme Bittner, fait de la force physique le cœur de la conception de la police. Quelques-uns, comme Brodeur, ont fait de l'illégalisme le point central de leur conception. Rares sont ceux qui, comme Manning, ont fait de la notion d'exception un point majeur de la définition de 
la police. Et, lorsqu'ils l'ont fait, ils ont hésité à ouvrir les perspectives théoriques que cette notion porte avec elle ${ }^{13}$. Quelles perspectives ouvre le recours à cette notion ?

Chez Schmitt, décider en situation exceptionnelle (c'est-à-dire : exercer la souveraineté) revient à exprimer le politique ; c'est-à-dire, dans l'esprit de Schmitt, poser la distinction ami / ennemi. En effet, ce n'est pas tant l'État qui est le produit de la décision souveraine, mais le politique lui-même. L'État n'est que la forme particulière, l'ingénierie que se donne "l'unité politique », la polity, par le geste même de la décision souveraine. Comme le souligne la fameuse déclaration, le concept d'État présuppose le concept du politique (Schmitt, 1934, 20), et le politique, l'unité politique, sont définis par ce moment particulier où l'ennemi est distingué de l'ami (1934, 22). La décision en situation exceptionnelle est le moment particulièrement intense où se manifeste le politique et cette manifestation est celle par laquelle un collectif, une société, identifie son ennemi propre. Selon cette interprétation, qui repose sur les premières pages de la Notion du politique du juriste allemand, la police, en tant qu'elle jouit d'espaces de souveraineté concédés par le système politique, est cette instance qui, dans nos sociétés rationnelles-légales, endosse le rôle de définir l'ennemi. La police est cette institution du quotidien (à la différence de l'armée et même de la justice pénale) qui se voit investie de perpétuer, dans l'État rationnel-légal, l'ordre normatif de procédure décrit par Max Weber (Colliot-Thélène, 2009), un État défini en substance ; en l'espèce défini par sa faculté de désigner l'ennemi au sein de la communauté. L'acte souverain établi par la police est, dans les espaces sociaux concernés, le geste par lequel la police fait d'un ensemble indistinct une communauté politique, une polity.

Mettons en garde, à ce moment de l'énoncé de cette hypothèse, contre une interprétation absolutiste de la notion d'ennemi. Schmitt avance une notion de l'ennemi apparemment tautologique, en tout cas procédurale : il n'y a pas d'essence préalable à l'ennemi, l'ennemi est celui qui est désigné comme tel. Avec le terme d'ennemi, Schmitt reprend (pour la subvertir - cf. Kervégan, 1992, 186) une notion par laquelle Hegel établit la communauté politique. Hegel (...) a donné une définition de l'ennemi, ce que les philosophes modernes évitent habituellement : il est la différence éthique (die sittliche Differenz), l'étranger à nier dans sa totalité vivante " (1934, 62). « L'étranger » n'est évidemment pas, sous peine d'anachronisme, à entendre en un sens stato-national, comme celui qui, présent sur le territoire national, l'est en tant que dépositaire d'une autre nationalité. Il est le stranger (der Fremde, sous la plume de Schmitt), et non le foreigner. L'étranger est celui, si l'on suit Hegel, qui ne partage pas l'éthique de la communauté, qui n'en partage pas les valeurs communes, tout simplement les mœurs (die Sitten). Si l'on suit ce raisonnement, la police moderne, dans les espaces de souveraineté qui lui sont accordés, est appelée à contrôler les indisciplinés, ceux dont les mœurs tranchent ou sont supposées trancher - avec les mœurs dominantes. Comme l'écrit Didier Fassin $(2011,314)$ dans son ethnographie récente d'un service de police, il s'agit d'ennemis (le terme est le sien) définis comme tels en ce qu'ils sont rejetés dans une altérité radicale ${ }^{14}$.

Saisir ce qui se présente lorsque l'on prend au sérieux l'idée selon laquelle l'illégalisme policier est au cœur de la notion de police, c'est-à-dire lorsque l'on examine les manifestations concrètes de cet illégalisme particulier, ouvre donc une dimension substantielle de la police. Cette dimension est celle du politique, de l'altérité éthique qui, à un moment particulier de son développement, définit les frontières et la nature de la polity. Comme si, en-deçà de l'État rationnel-légal, la police ouvrait pour la perpétuation de sa dimension substantielle, sa dimension 
irréductiblement politique. Ce qui, très vraisemblablement, explique son irréductible violence. Mais mesurer toutes les conséquences théoriques de la définition avancée par Jean-Paul Brodeur dans The Policing Web occuperait bien plus d'espace qu'on ne peut le faire dans le cadre de cet hommage - qu'il nous faut à présent conclure.

\section{Conclusion}

Avec les notions de polity, de souveraineté ou d'ennemi, nul doute que les recherches les plus empiriques (pour ne pas dire empiricistes) sur la police s'articuleraient bien mieux à divers champs de la théorie politique qu'elles ne le font à présent - si tant est qu'elles aspirent à quelque lien que ce soit avec quelque théorie que ce soit. Toutes les recherches, parvenues à un degré très avancé de cumulativité, portant sur la relation entre le racial/minority threat et la police, qui suggèrent que le degré de police (le nombre de policiers ou le budget des départements de police) est indexé au nombre de Noirs dans la ville, trouveraient là très vraisemblablement des débouchés théoriques prometteurs.

Il faut toutefois rappeler combien conférer a priori une dimension raciale à l'ennemi sur-simplifierait le problème pour en étouffer la dimension heuristique. Il est d'ailleurs curieux de constater, de ce point de vue, le rejet de la dimension schmittienne de la police chez Jean-Paul Brodeur et chez son collègue Egon Bittner, avec lequel Brodeur entretint des échanges fructueux au plus haut degré. Voici ce que dit Bittner lors de ces échanges :
Finally, I want to disregard the very influential formulation of the function of the state by Carl Schmitt, namely that the state is defined by the presence of its enemies. And now, what is left is essentially what was present already in the city of antiquity. That is, it's a polis that already redefines the minimal state, where it literally consists of the organization of conditions of urban life. (...) What then the state provides in its minimal function is creating conditions for the orderly coexistence of strangers.(Bittner, in Brodeur 2007, 111)

Ce passage interroge tout particulièrement le lecteur car on y voit comment Egon Bittner (avec l'assentiment manifeste de son interlocuteur) refuse de reconnaître à la police son véritable statut dans la constitution des communautés politiques, des polities. Certes, comme le dit Bittner, la police est en charge d'assumer l'ordre interne de la ville, qui consiste en la coexistence pacifique de communautés étrangères les unes aux autres. Non pas étrangères au sens où elles relèveraient de nations ou de pays différents, mais étrangères au sens où les mœurs qui animent chacune d'elles sont étrangères les unes aux autres, et que la vie harmonieuse dans un même cadre urbain exige l'imposition d'une discipline, d'une éthique par un des groupes. Ce ne sont pas les Noirs, les étrangers (foreigners), les Maghrébins, mais les men without roots (Emsley, 2007, 43), les assholes (Van Maanen, 1978), ou plus tautologiquement la police property (Lee, 1981) qui contribuent, par les actes souverains de la police, à forger la polity. L'imposition, à leurs dépens, d'une discipline (Choongh, 1997) est l'acte de baptême, chaque fois renouvelé, de la communauté politique. Là encore, le policier juge du mandat politique dont il estime être dépositaire et, la société changeant, le mandat change avec elle. Il n'est que penser aux activités de la « Morals Division » du New York Police Department, fort occupées par la chasse aux homosexuels... chasse qui donna naissance à la mobilisation de Stonewall et, en bout de course, au changement complet de mandat politique concernant la place des homosexuels dans la communauté politique. À l'inverse, ce n'est que passées des décennies d'indifférence que les policiers du Los Angeles Police Department ont désigné / se sont vus désigner les migrants mexicains comme leur ennemi, contribuant ainsi, au fil des années, à forger l'identité politique de ces migrants dans la communauté politique américaine ou, à tout le moins, 
californienne (Escobar, 1999). L'économie de la force ou de la retenue, estimée, voire délibérée par les policiers au cours de l'interaction, est la traduction dans l'action du mandat politique (voir dans le même sens Favre, 2010, 1241-43).

Jean-Paul Brodeur ne dit pas les choses autrement, dans la définition qu'il donne de la police, dont le rôle est de enforce various types of rules and customs that promote a defined order in society. Nous dirons pour notre part qu'il s'agit autant de définir un ordre public (comme l'ensemble des règles de vie commune) que de contribuer à la définition substantielle du politique. C'est du reste ce qui rend, au sens fort du terme, l'institution policière si polémique : elle est au cœur du politique. C'est aussi ce qui rend extrêmement difficile toute entreprise visant son amendement ou sa réforme : quel rôle confier à la police si ce n'est celui de contrôler les marges de l'ordre social et de les civiliser?

Pour autant, rappelons que les policiers, pour exercer des actes de souveraineté (entendus au sens où nous les avons définis), ne sont pas affranchis de toute règle. Ils sont, au contraire, pris dans des contraintes qui tiennent à la nature de leur interlocuteur, à la nature du lieu, des personnes présentes, mais aussi des circonstances et de la conjoncture politique - de ce qui se déroule sur le plan de la politics. La police discretion n'est pas donnée a priori, elle est évaluée dans l'action par les policiers eux-mêmes, qui estiment ce que la communauté politique est prête à assumer et ce qu'elle rejettera comme n'étant plus de l'illégalisme policier, mais de l'infraction pure. Le mandat politique conféré à la police n'est pas donné a priori, intangible ; il est éprouvé par les policiers à chacune des interactions auxquelles ils se livrent.

Aussi, pour reprendre à notre tour, après Jean-Paul, la définition que donne Egon Bittner de la police, nous dirions (en soulignant les différences) :

The role of the police is best understood as a mechanism for the distribution of non-negotiably means employed in accordance of the dictates of an intuitive grasp of the political exigencies.

Où « politique » renvoie dans le même temps à l'acte d'évaluation par les policiers de l'ampleur du mandat que leur confie la communauté, et à l'effet propre, structurant et substantiel, de leur action sur la nature et les délimitations de la polity. On le voit : la police ne se situe pas dans l'ordre de la simple civilité, mais au fondement de nos sociétés politiques - comme si, au fond, il revenait à la police le mandat politique de faire contrepoids à la froide rationalité de l'État et au désenchantement du monde.

\section{Bibliographie}

Akrich M., 1992, The de-scription of technical objects, in Bijker W., Law J. (Eds), Shaping Technology/Building Society, Cambridge, MIT Press, 205-224.

Anderson M., 2011, In Thrall to Political Change: Police and Gendarmerie in France, Oxford, Oxford University Press.

Bittner E., 2001, Florence Nightingale à la poursuite de Willie Sutton, Déviance et Société, 25, 3, 285-306 (trad. de l'article paru en 1974, in Jacob (dir.), 1974, The Potential for Reform of Criminal Justice).

Bittner E., Brodeur J.-P., Jobard F., Lévy R., 2001, Débat. Autour de Bittner, Déviance et Société, 25, 3, 279-345.

Black D., 1973, The Mobilization of Law, The Journal of Legal Studies, 2, 1, 125-149.

Bourdieu P., 2012, Sur l'État. Cours au Collège de France. 1989-92, Paris, Seuil.

Brodeur J.-P., (ed.), 1983, High and Low Policing. Remarks on the Policing of Political Activities, Social Problems, 30, 5, 507-520 (repris et révisé in La police politique. La haute police, in Les visages de la police. Pratiques et perceptions, Montréal, Presses universitaires de Montréal, 2003, 225-254).

Brodeur J.-P., (dir.), 2005, La police en pièces détachées, Criminologie, 38, 2, 3-172. 
Brodeur J.-P., 2011, The Policing Web, Oxford, Oxford University Press.

Brodeur J.-P., 2008, Que dire maintenant de la police ?, in Monjardet D., Chauvenet A., Ocqueteau F., (dir.), Notes inédites sur les choses policières (1999-2006), Paris, La Découverte, 255-268.

Brodeur J.-P., 2008, Haute et basse police après le 11 septembre, Criminologie, 41, 1, 133-151 (repris in Jean-Paul Brodeur d'hier à aujourd'hui, numéro spécial de Criminologie, 44, 1, 2011, p. 225-245, présentation F. Ocqueteau).

Bugnon G., 2011, Le constat médical peut-il mettre à l'épreuve les frontières de la force policière légitime? Enquête sur un dispositif médico-légal de dépistage des violences policières, Déviance et Société, 35, 1, 113-136.

Cahn O., 2007, Actualité de l'arrêt R. v. Secretary of State for the Home Department ex parte Ramda du 27 juin 2002 : incident isolé ou précédent dommageable ? Cultures \& Conflits, 66, 121-156.

Choongh S., 1997, Policing as social discipline, Oxford, Clarendon Press.

Colliot-Thélène C., 1999, Carl Schmitt versus Max Weber: Juridical Rationality and Economic Rationality, in Mouffe Ch., The Challenge of Carl Schmitt, Londres, Verso Books.

Colliot-Thélène C., 2009, Modern rationalities of the political. From Foucault to Weber, Max Weber Studies, 9, 1-2, 165-187.

Cornevin Ch., 2010, Plus de 3100 policiers sanctionnés en 2009, Le Figaro, 16 juillet.

Douglas M., 1966, Purity and Danger. An Analysis of Concept of Pollution and Taboo,Londres, Routledge \& Kegan Paul.

Dulong R., 1997, Les opérateurs de factualité. Les ingrédients matériels et affectuels de l'évidence historique, Politix, 10, 39, 65-85.

Dulong R., 1998, Le témoin oculaire. Les conditions sociales de l'attestation personnelle, Paris, Éditions de l'EHESS.

Emsley C., 2007, Crime, police, and penal policy: European Experiences 1750-1940, Oxford, Oxford University Press.

Escobar E.J., 1999, Race, police, and the making of a political identity: Mexican Americans and the Los Angeles Police Department, 1900-1945, Berkeley, University of California Press.

Fassin D., 2011, La force de l'ordre. Une anthropologie de la police des quartiers, Paris, Seuil.

Favre P., 2010, Quand la police fabrique l'ordre social. Un en-deçà des politiques publiques de la police? Revue française de science politique, 59, 6, 1231-1250.

Fischer N., 2011, « Guerre aux migrants ? ». Le modèle du camp à l'épreuve de l'économie du maintien de l'ordre et de la subversion en rétention administrative, contribution au XIe Congrès de l'Association Française de Science Politique, Strasbourg, section thématique $n^{\circ} 9$. Section thématique 9, « Ni guerre, ni paix : enquêtes sur les ordres politiques disloqués ».

Fischer N., Spire A., 2009, L'État face aux illégalismes, Politix, 22, 3, 7-20.

Hodgson J., 2002, Suspects, defendants and victims in the French criminal process. The context of recent reform, International and Comparative Law Quarterly, 51, 4, 781-816.

Jobard F., 2003, Raw facts and narratives, Policing and Society, 13, 4, 423-428.

Jobard F., 2005, Le nouveau mandat policier. Faire la police dans les zones dites 'de non-droit', Criminologie, 38, 2, 103-121.

Jobard F., Linhardt D., 2008, Control and guardianship. A comparison between two surveillance spaces, Orly international airport and Dammarie's housing project, in Deflem M. (Ed.), Surveillance and Governance. Crime Control and Beyond, Bingley, Emerald Pbg/JAI Press, 77-102.

Klockars C.B., 1985, The Idea of Police, California, Sage Publications Beverly Hills.

Ker Muir W., 1977, Police. Streetcorner Politicians,Chicago, Chicago University Press.

Kervégan J.-F., 1992, Hegel, Carl Schmitt. Le politique entre spéculation et positivité, Paris, Presses universitaires de France.

Krynen J., 2012, L'état de justice. France, XIIIe-XXe siècles, Paris, Gallimard, vol. 2.

Lagrange H., 2009, The French riots and segregation, in Waddington D., Jobard F., King M. (Eds), Rioting in the UK and France. A Comparative Analysis, Cullompton, Wilan, 107-123.

Le Doussal P., 1988, Étude sur les fautes disciplinaires, Paris, IGPN, ministère de l'Intérieur.

Lee J., 1981, Some structural aspects of police deviance in relations with minority groups, in Shearing C., Stenning Ph. (Eds), Organizational Police Deviance. Its Structureand Control, Toronto, Butterworths, 49-82. 
Manning P.K., 1977, Rules, Colleagues, and Situationally Justified Actions, in Manning P.K., Van Maanen J. (Eds), Policing. A View from the Street, New York, Random House, 71-88.

Manning P.K., 2003, Policing Contingencies, Chicago, University of Chicago Press.

Manning P.K., 2010, Democratic policing in a changing world, Boulder, Paradigm Publishers.

McHeyman, J.M.C., 200o. Respect for outsiders? Respect for the law? The moral evaluation of high scale issues by US immigration officers, Journal of the Royal Anthropological Institute, 6, 4, 635-652.

Moreau de Bellaing C., 2009, Violences illégitimes et publicité de l'action policière, Politix, 22, 3, 119-141.

Müller J.W., 2003, A Dangerous Mind. Carl Schmitt in Post-War European Thought, Yale, Yale University Press.

Ocqueteau F., 2004, Polices entre État et marché, Paris, Presses de Sciences Po.

Reiner R., 1992, The Politics of the Police, Toronto, Toronto University Press, $2^{\mathrm{d}}$ ed.

Reiss A.J., Bordua D.J., 1967, Environment and Organization. A Perspective on the Police, in Bordua D.J. (Ed.), The Police. Six Sociological Essays, New York, John Wiley \& Sons, 25-54.

Rodley N., 2005, Torture, Violence, and the Global War on Terror, American Society of International Law Proceedings, 99, 402-407.

Salvy E., 2012, MPs table questions over French 'President's Spy' revelations, Mediapart, 26 janvier.

Schmitt C., 1932, Der Begriff des Politischen, Berlin, Duncker \& Humblot (trad. angl. : The Concept of the Political, transl. G. Schwab, Chicago, Chicago University Press, 2007).

Schmitt C., 1934, Politische Theologie. Vier Kapitel zur Lehre von der Souveränität, Berlin, Duncker \& Humblot (trad. gb : Political Theology. Four Chapters on the Concept of Sovereignty, transl. G. Schwab, Cambridge, MIT Press).

Skogan W.G., Frydl K., 2004, Fairness and Effectiveness in Policing. The evidence, Washington, The National Academies Press.

Stenning Ph., 2000, Evaluating Police Complaints Legislation. A Suggested Framework, in Goldsmith A., Lewis C. (Eds), Civilian Oversight of Policing. Governance, Democracy and Human Rights, Oxford, Hart, 147-163.

Uildriks N., 1999, Police torture in France, Netherlands Quarterly of Human Rights, 17, 4, 411-423.

Van Maanen J., 1978, The asshole, in Manning P., Van Maanen J. (Eds), Policing. A view from the street, New York, Random House, 221-237.

Willsher K., 2011, French intelligence chief accused of spying on journalist, The Guardian, 10 octobre.

\section{Notes}

1 Voir le dossier dirigé par René Lévy de la revue Déviance et Société (2001) consacré à l'apport d'Egon Bittner à la sociologie de la police, ou l'invitation qui m'avait été faite par Jean-Paul de contribuer, avec d'autres jeunes chercheurs français, au numéro anniversaire de la revue canadienne Criminologie (2005), numéro anniversaire de la fameuse édition de 1984 sur la police, qu'il avait dirigée.

2 À l'attention des lecteurs étrangers qui connaîtraient mal la situation française en la matière, signalons au rang des événements publiés par la presse nationale au cours du seul mois de janvier 2012 (presse nationale qui dressait un bilan de 14 policiers et gendarmes décédés en service en 2011) : la mort au cours d'une interpellation d'un jeune homme de 30 ans, Wissan El-Yamni (vraisemblablement par étouffement) ; l'acquittement par la Cour d'assises de Nice de 4 policiers jugés pour viol d'une prostituée ; la confirmation par un jugement du Tribunal administratif de la mise à la retraite d'office d'un policier qui avait publié les fiches policières de deux célébrités françaises pour protester contre la tenue de telles fiches (qu’il jugeait contraires aux libertés individuelles) ; la commutation en sursis d'une peine de prison prononcée en 2011 contre des policiers ayant sur la base de faux accusé un individu de tentative d'homicide contre des policiers ; l'appel à manifester d'un syndicat de police contre l'apposition d'une plaque par une municipalité de banlieue parisienne commémorant le décès d'un administré, Ali Ziri, 69 ans, à l'issue de sa garde à vue dans les locaux du commissariat de la ville.

3 Notre «traduction » française est une trahison assumée, qui arrime le terme à l'héritage foucaldien. Pour Michel Foucault, à l'image de la fraude fiscale, l'illégalisme est ce contournement de la loi qui est nécessaire à la perpétuation de l'ordre social : L’illégalisme 
n'est pas un accident, une imperfection. C'est un élément absolument positif du fonctionnement social. Tout dispositif législatif a ménagé des espaces protégés et profitables où la loi peut être violée, d'autres où elle peut être ignorée, d'autres, enfin, où les infractions sont sanctionnées (Foucault 1976, cité in Fischer, Spire, 2009, 86). Curieusement, Brodeur ne fait quasiment pas référence à Foucault dans The Policing Web.

4 Le participe présent policing contribue, avec le pluriel de « agents ", à la restitution de la pluralité des acteurs et des actions : In line with my inventory of persons professionally involved in policing, I prefer the expression 'policing agent' to the word 'police' which generally refers to public constabularies (Policing Web, 130). Voir l'importation de ce participe présent chez Ocqueteau, 2004, 50.

5 C'est d'ailleurs comme promoteur d'une définition duale de la police, partagée entre " haute » et " basse » police, que Jean-Paul Brodeur a affirmé son prestige dans le monde anglo-saxon (Brodeur 1983/2003, 2008/2011).

6 Là aussi, l'actualité française est prolixe (Willsher, 2011 ; Salvy, 2012).

7 Voir de manière plus générale la prétention des juges de « créer » la loi en fonction de ce qu'ils perçoivent de son adéquation à ce que la société attend d'elle (Krynen, 2012, 210).

8 Sur ce caractère insuffisant en soi, des attestations médicales, voir sur le cas français la démonstration empirique récente de Bugnon, 2011 et les observations de Moreau de Bellaing, 2009, 87-89 notamment.

9 Contrairement aux procédures de common law, la loi française ne prévoyait pas la présence de l'avocat en garde à vue, du moins avant la réforme du Code de procédure pénale de janvier 1993 (Hodgson, 2002) - réforme engagée du reste à la suite de décès en cellule de police, tandis que celle qui la compléta (loi du 15 juin 2000) fut en partie la conséquence de la condamnation de la France pour torture dans l'affaire Selmouni.

10 Voir pour une démonstration semblable, à partir des dossiers disciplinaires parisiens, Moreau de Bellaing, 2009.

11 Le service chargé d'enquêter sur les allégations de fautes par les fonctionnaires de police (Anderson, 2011, 395). Le rapport fut non publié, en dépit du fait qu'il fut commandé par le ministre de l'Intérieur de l'époque.

12 Selon le directeur du service d'inspection de la Police nationale, l'IGPN, les policiers sont destinataires de 50\% des sanctions prononcées contre les fonctionnaires en France, alors qu'ils ne constituent que $8 \%$ de la fonction publique de ce pays (Boyajean in Cornevin, 2010). De ce point de vue, les policiers font l'objet d'un contrôle resserré. Mais cette voie administrative, disciplinaire, voire collégiale de règlement des déviances est une chose, la justice publique de la force publique en est une autre (Stenning, 2000).

13 Peter K. Manning (2003 et surtout 2010, 68) propose une définition multidimensionnelle de la police, qui mentionne l'exception comme un point de fuite : The police as an organization in Anglo-American societies, constituted of many diverse agencies, are authoritatively coordinated, legitimate organizations. They stand ready to apply force up and including fatal force in politically defined territories. They seek to sustain politically defined order and ordering via tracking, surveillance and arrest. As such, they require compliance to command from lower personnel and citizens and the ability to proceed by exception. Si l'on voit combien le politique est central dans cette définition (ce qui en fait une définition originale), l'exception est plus le point de fuite que la pierre de touche de la notion de police. Manning n'accorde que 8 lignes à cette notion, soulignant combien cette dimension has not been well explored, although political theorists, notably Carl Schmitt, have written about the importance of 'exceptionalism' in the face of crises (Manning 2010, 79-80).

14 Relevons que cette ethnographie, qui fait appel à la notion d'exception (« petit état d'exception ») en la rattachant à Walter Benjamin (Fassin, 2011, 330), vise une « anthropologie de l'État », dans une perspective plus soucieuse de la dimension postcoloniale de l'action de l'État que de la théorie de l'État en elle-même.

\section{Pour citer cet article}

Référence électronique

Fabien Jobard, «Propositions sur la théorie de la police », Champ pénal/Penal field [En ligne], Vol. IX | 2012, mis en ligne le 12 mai 2012, Consulté le 22 octobre 2012. URL :

http://champpenal.revues.org/8298 ; DOI : 10.4000/champpenal.8298

\section{Auteur}


Directeur du CESDIP (UMR 8183, CNRS/UVSQ, ministère de la Justice).

Contact : fabjob@cesdip.fr

Articles du même auteur

Proposition on the theory of policing [Texte intégral]

Paru dans Champ pénal/Penal field, Vol. IX | 2012

\section{Droits d'auteur}

(C) Champ pénal 